\title{
Contribution of Higher-Order Dispersion to Nonlinear Dust Ion Acoustic Waves in Inhomogeneous Mesospheric Dusty Plasma with Dust Charge Fluctuation
}

\author{
Mohamed T. Attia, Mohsen A. Zahran, Emad K. El-Shewy, and Ahmed E. Mowafy \\ Theoretical Physics Group, Faculty of Science, Mansoura University, Mansoura, Egypt \\ Reprint requests to E. K. E.-S.; E-mail: e_k_el_shewy@mans.edu.eg or emadshewy@yahoo.com
}

Z. Naturforsch. 65a, 91 - 99 (2010); received November 17, 2008 / revised March 25, 2009

\begin{abstract}
The propagation of dust ion acoustic waves (DIAWs) in a weakly inhomogeneous, weakly coupled, collisionless, and unmagnetized four components dusty plasma are examined. The fluid system considered in this work consists of cold positive ions, cold negatively and positively charged dust particles associated with isothermal electrons. For nonlinear (DIAW) waves, a reductive perturbation method was employed to obtain the variable coefficients Kortewege-de Vries (KdV) equation for the first-order potential. For local inhomogenity, the present system admits the coexistence of rarefactive and compressive solitons. As a matter of fact, when the wave amplitude enlarged, the width and velocity of the wave deviate from the prediction of the $\mathrm{KdV}$ equation. It means that we have to extend our analysis to obtain the variable coefficients Kortewege-de Vries (KdV) equation with fifth-order dispersion term. For locally constant parameters, the higher-order solution for the resulting equation has been achieved via what is called perturbation technique. The effects of positive and negative dust charge fluctuations on the higher-order soliton amplitude and width of electrostatic solitary structures are outlined.
\end{abstract}

Key words: Inhomogeneous Dusty Plasma; Dust Ion Acoustic Waves; Positive and Negative Dust Charge Fluctuation; Variable Coefficients KdV Equation; Higher-Order Solution.

\section{Introduction}

The study of the dynamics of dust contaminated plasmas (mixtures of ordinary plasma particles and charged dust grains) has recently received considerable interest due to their occurrence in real charged particle systems, e. g., in interstellar clouds, in interplanetary space, in cometary tails, in ring systems of giant planets, in mesospheric noctilucent clouds, as well as in many Earth bound plasma [1 -2] and later experimentally confirmed [3].

The presence of highly negatively charged and massive grains of dust particles in an electron ion plasma is responsible for the appearance of new types of waves, depending on whether the dust grains are considered to be static or mobile. One type of these waves is the dust ion acoustic wave (DIAW) which is the usual ion acoustic wave modified by the presence of dust grains. After the theoretical prediction of the existence of DIAWs by Shukla and Siline [4] and their experimental observation by Barkan et al. [5]. It was noted that in studying collective effects involving charged dust particles in dusty plasmas, one generally assumes that the dust grains behave like point charges. In fact, the dust particles immersed in a plasma are not neutral, but they act as probes and collect the background plasma electrons and ions. Since ions are much heavier than electrons, it turns out that the dust grains immersed in a gaseous plasma are usually negatively charged.

There are some dust grains charging mechanisms, namely secondary electrons, thermoionic emission, photoelectron emission by UV radiation, field emission, radioactivity, impact ionization, etc. [6]. Furthermore, there are a direct evidence for the existence of both positively and negatively charged dust particles, for instance in space environments, such as, cometary tails and mesosphere [6-9], and also in laboratory plasma [10].

Noticeably, most of the studies have been done for the homogeneous plasmas, whereas inhomogeneity exists widely in space and laboratory discharges [11]. Inhomogeneity may stem from density and/or temperature gradient or it could be due to magnetic field in space. It is much more appreciable near to the edges and the boundaries of the system [12]. The effect of inhomogeneity of dusty plasma on the propagation of 
waves is an another important problem from various points of view. In [13], El-Wakil et al. studied the effect of the presence of negative and positive dust grains on the propagation of the DIAWs in inhomogeneous mesospheric dusty plasma. Later, Mowafy et al. [14] investigated the effect of positive and negative dust charge fluctuation on the propagation of DIAWs in a weakly inhomogeneous, weakly coupled, collisionless, and unmagnetized mesospheric dusty plasma consisting of a four components dusty plasma.

Investigations of small amplitude DIAWs in inhomogeneous mesospheric dusty plasma usually describe the evolution of the wave by the variable coefficient Korteweg-de Vries (KdV) equation. In fact, this equation contains the lowest-order nonlinearity and dispersion, and consequently can only describe a wave restricted to a small amplitude. In other words, the firstorder solution would underestimate the amplitude of the solitary wave by as much as $20 \%$. As the wave amplitude increases, the width and velocity of a soliton deviate from the prediction of the $\mathrm{KdV}$ equation, i. e. the KdV approximation does not apply anymore. Therefore, in order to overcome this deviation, higherorder nonlinear and dispersive effects have to be taken into account [15-21].

To the author's knowledge, the effects of higherorder dispersion corrections to the propagation of DIAWs in inhomogeneous dusty plasmas have not been addressed in the literature before. So, our motive here is to study the effect of the higher-order dispersion term on the propagation velocity, the amplitude, and the width of the soliton solution in a weakly inhomogeneous, weakly coupled, collisionless, and unmagnetized four components dusty plasma.

This paper is organized as follows: In Section 2 we present the basic set of fluid equations governing our plasma model. In Section 3 we derive the KdV equation with lowest-order nonlinearity and dispersion for describing nonlinear DAWs. In Section 4, a modified $\mathrm{KdV}$ equation incorporating the fifth-order dispersion term is introduced and its higher-order solution is obtained. Finally, discussions and conclusions are given in Sections 5 and 6.

\section{Governing Equation and Derivation of the Variable Coefficient KdV Equation}

We consider a fully ionized, weakly inhomogeneous, weakly coupled, collisionless, and unmagnetized plasma consisting of cold positive ions, cold negatively and positively charged dust particles, and isothermal electrons. The basic equations describing the system is given in dimensionless variables by Mowafy et al. [14].

For positive ions, it is

$$
\begin{aligned}
& \frac{\partial}{\partial t} n_{\mathrm{i}}+\frac{\partial}{\partial x}\left(n_{\mathrm{i}} u_{\mathrm{i}}\right)=0, \\
& \left(\frac{\partial}{\partial t}+u_{\mathrm{i}} \frac{\partial}{\partial x}\right) u_{\mathrm{i}}+\frac{\partial}{\partial x} \phi=0 .
\end{aligned}
$$

The equation for positive dust grains charging takes the form

$$
\frac{\partial Z_{\mathrm{p}}}{\partial t}=\frac{I_{\mathrm{p}}+I_{\mathrm{e}}^{-}}{e}
$$

with photo-emission current and electron absorption current given by

$$
I_{\mathrm{p}}=P_{1} \exp \left(-P_{2} Z_{\mathrm{p}}\right), \quad I_{\mathrm{e}}^{-}=-\left(1+P_{2} Z_{\mathrm{p}}\right) P_{3} n_{\mathrm{e}},
$$

while the equation required for negative dust grains charging becomes

$$
-\frac{\partial Z_{\mathrm{n}}}{\partial t}=\frac{I_{\mathrm{i}}+I_{\mathrm{e}}}{e},
$$

with ion current and electron current given by

$$
\begin{aligned}
& I_{\mathrm{e}}=-N_{1} n_{\mathrm{e}} \exp \left(N_{3} Z_{\mathrm{n}}\right), \\
& I_{\mathrm{i}}=N_{2} n_{\mathrm{i}}\left[F_{1}\left(u_{0}\right)+F_{2}\left(u_{0}\right) \cdot N_{4} Z_{\mathrm{n}}\right]
\end{aligned}
$$

and finally concerning the isothermal electrons

$$
n_{\mathrm{e}}=\exp (\phi) .
$$

Obviously, these equations are coupled through Possion's equation as

$$
\frac{\partial^{2}}{\partial x^{2}} \phi=-n_{\mathrm{i}}+Z_{\mathrm{n}}^{(0)} Z_{\mathrm{n}} n_{\mathrm{n}}-Z_{\mathrm{p}}^{(0)} Z_{\mathrm{p}} n_{\mathrm{p}}+n_{\mathrm{e}}
$$

where $P_{1-3}, N_{1-4}$ and $F_{1,2}\left(u_{0}\right)$ are listed in the Appendix.

In (1)-(6), $n_{\mathrm{i}}, n_{\mathrm{n}}, n_{\mathrm{p}}$, and $n_{\mathrm{e}}$ are the densities of ions, negative dust grains, positive dust grains, and electrons, respectively. The velocity of ions is represented by $u_{\mathrm{i}} ; u_{0}$ is the ratio of streaming velocity to the ion thermal speed; $m_{\mathrm{e}}\left(m_{\mathrm{i}}\right)$ and $T_{\mathrm{e}}\left(T_{\mathrm{i}}\right)$ are the mass and the temperature of electrons (ions), respectively. $K_{\mathrm{B}}$ is the Boltzmann constant. $Z_{\mathrm{p}}\left(Z_{\mathrm{n}}\right)$ denotes to the positive (negative) dust grain charge number. $C\left(r_{\mathrm{d}}\right)$ is 
the capacitance (radius) of the spherical dust grains, $J$ is the UV photon flux, $Y$ is the yield of photons. $\phi$ is the electric potential, $x$ is the space coordinate, and $t$ is the time variable. All these quantities are dimensionless, being normalized in terms of the following characteristic quantities: $n$ by the unperturbed electron density $n_{\mathrm{e}}^{(0)}, u$ by the ion sound velocity $\left(K_{\mathrm{B}} T_{\mathrm{e}} / m_{\mathrm{i}}\right)^{1 / 2}$, $\phi$ by $\left(K_{\mathrm{B}} T_{\mathrm{e}} / e\right), t$ by the inverse of the plasma frequency $\omega_{\mathrm{pi}}^{-1}=\left(m_{\mathrm{i}} / 4 \pi e^{2} n_{\mathrm{i}}^{(0)}\right)^{1 / 2}, x$ by the electron Debye length $\lambda_{\mathrm{d}}=\left(K_{\mathrm{B}} T_{\mathrm{e}} / 4 \pi e^{2} n_{\mathrm{i}}^{(0)}\right)^{1 / 2}$ and $Z_{\mathrm{p}}\left(Z_{\mathrm{n}}\right)$ by the unperturbed number of charges residing on the dust grains $Z_{\mathrm{p}}^{(0)}\left(Z_{\mathrm{n}}^{(0)}\right)$.

The charge neutrality at equilibrium requires that

$$
\delta_{\mathrm{i}}-Z_{\mathrm{n}}^{(0)} \delta_{\mathrm{n}}+Z_{\mathrm{p}}^{(0)} \delta_{\mathrm{p}}-1=0,
$$

where $\delta_{\mathrm{i}}=n_{\mathrm{i}}^{(0)} / n_{\mathrm{e}}^{(0)}, \delta_{\mathrm{n}}=n_{\mathrm{n}} / n_{\mathrm{e}}^{(0)}$, and $\delta_{\mathrm{p}}=n_{\mathrm{p}} / n_{\mathrm{e}}^{(0)}$ denote the ion, negative dust grains and positive dust grains densities to electron density ratio(s), respectively. To study the dynamics of small but finite amplitude DIAWs, we derive the $\mathrm{KdV}$ equation from the basic set of equations (1)-(6), by employing the reductive perturbation technique as well as the stretched coordinates applicable for spatially inhomogeneous plasma [22].

We obtain

$$
\zeta=\varepsilon^{1 / 2}\left(\int \frac{\mathrm{d} x}{\lambda_{0}(x)}-t\right) \text { and } s=\varepsilon^{3 / 2} x,
$$

where $\varepsilon$ is a smallness parameter measuring the weakness of the amplitude or dispersion and $\lambda_{0}(x)$ is the soliton velocity. We can expand the variables $n_{\mathrm{i}}, Z_{\mathrm{n}}$, $Z_{\mathrm{p}}$, and $\phi$ about the unperturbed states in power series of $\varepsilon$ as the following:

$$
\left(\begin{array}{c}
n_{\mathrm{i}} \\
u_{\mathrm{i}} \\
Z_{\mathrm{n}} \\
Z_{\mathrm{p}} \\
\phi
\end{array}\right)=\left(\begin{array}{c}
\delta_{\mathrm{i}} \\
u_{\mathrm{i}}^{(0)} \\
Z_{\mathrm{n}}^{(0)} \\
Z_{\mathrm{p}}^{(0)} \\
\phi^{(0)}
\end{array}\right)+\left(\begin{array}{c}
\varepsilon n_{\mathrm{i}}^{(1)}+\varepsilon^{2} n_{\mathrm{i}}^{(2)}+\ldots \\
\varepsilon u_{\mathrm{i}}^{(1)}+\varepsilon^{2} u_{\mathrm{i}}^{(2)}+\ldots \\
\varepsilon Z_{\mathrm{n}}^{(1)}+\varepsilon^{2} Z_{\mathrm{n}}^{(2)}+\ldots \\
\varepsilon Z_{\mathrm{p}}^{(1)}+\varepsilon^{2} Z_{\mathrm{p}}^{(2)}+\ldots \\
\varepsilon \phi^{(1)}+\varepsilon^{2} \phi^{(2)}+\ldots
\end{array}\right)
$$

Inserting the stretching (8) and the expansions (9) into the basic equations (1) - (6) gives a series of equations, upon separating out the different orders in $\varepsilon$.

Since, we are considering only spatial gradients, it follows that

$$
\frac{\partial}{\partial \zeta} \delta_{i}=\frac{\partial}{\partial \zeta} \lambda_{0}(x)=0
$$

In equilibrium (1) to (6), one can get the relations

$$
\frac{\partial}{\partial s}\left(\delta_{\mathrm{i}} u_{\mathrm{i}}^{(0)}\right)=0, u_{\mathrm{i}}^{(0)} \frac{\partial}{\partial s} u_{\mathrm{i}}^{(0)}+\frac{\partial}{\partial s} \phi^{(0)}=0 .
$$

Second-order in $\varepsilon$, together with the aid of zero- and first-order equations, gives the nonlinear partial differential equation [13]

$$
\begin{aligned}
& \frac{1}{2 \lambda_{0} \delta_{\mathrm{i}} D_{1}} \frac{\partial^{3}}{\partial \zeta^{3}} \phi^{(1)}+\frac{\lambda_{0} D_{2}}{2 \delta_{\mathrm{i}} D_{1}} \phi^{(1)} \frac{\partial}{\partial \zeta} \phi^{(1)} \\
& +\frac{\partial}{\partial s} \phi^{(1)}-\frac{\phi^{(1)}}{2 \lambda_{0}} \frac{\partial}{\partial s} \lambda_{0}+\frac{\phi^{(1)}}{2 \delta_{\mathrm{i}}} \frac{\partial}{\partial s} \delta_{\mathrm{i}}=0,
\end{aligned}
$$

where $D_{1}$ and $D_{2}$ are given in the Appendix.

For (12), it has been assumed that the equilibrium flow velocity of ions $u_{\mathrm{i}}^{(0)}$ is neglected by comparing it with DIAW phase speed $\lambda_{0}$. Hence, in order to obtain the solitary wave solutions, one can recast (12) via the following transformation, $\Psi=\left(\frac{\delta_{\mathrm{i}}}{\lambda_{0}}\right)^{1 / 2} \phi^{(1)}$, to get the well-known $\mathrm{KdV}$ equation

$$
\frac{\partial}{\partial s} \Psi+A \Psi \frac{\partial}{\partial \zeta} \Psi+\frac{B}{2} \frac{\partial^{3}}{\partial \zeta^{3}} \Psi=0
$$

where the constants $A$ and $B$ are considered to be

$$
A=\left(\frac{\lambda_{0}^{5}}{\delta_{\mathrm{i}}}\right)^{1 / 2} \frac{D_{2}}{2 \lambda_{0} \delta_{\mathrm{i}} D_{1}}, \quad B=\frac{1}{\lambda_{0} \delta_{\mathrm{i}} D_{1}} .
$$

Remarkably, it has been thought that the nonlinear coefficients functionally depend on the space of the plasma, but for sake of simplicity for mathematical development, the variations are assumed to be negligibly small as compared to the characteristic scale length or the parameters could be locally constant. It means that (13) admits the hall-mark soliton solution

$$
\Psi=\frac{3 \vartheta}{A} \operatorname{sech}^{2}\left[\left(\frac{\vartheta}{2 B}\right)^{1 / 2} \eta\right]
$$

where $\eta$ is the transformed coordinate with respect to a frame moving with velocity $\vartheta$. It is important and worthful to remark that our system supports two kinds of potential structure, namely compressive and rarefactive. Depending on the sign of the coefficient of the nonlinear term $(A)$, compressive soliton exists if $A>0$ while rarefactive soliton exists if $A<0$. 


\section{Higher Order Correction}

As is well know, (13) contains the lowest-order nonlinearity and dispersion, and consequently the validity of it is restricted to waves of only small amplitudes. However, as the wave amplitude increases, the width and velocity of a soliton deviate from the prediction of the $\mathrm{KdV}$ equation. In particular, we add to the wellknown $\mathrm{KdV}$ equation a fifth-order dispersion term as a higher-order perturbation term

$$
\frac{\partial}{\partial s} \Psi+A \Psi \frac{\partial}{\partial \zeta} \Psi+\frac{B}{2} \frac{\partial^{3}}{\partial \zeta^{3}} \Psi+\varepsilon \frac{\partial^{5}}{\partial \zeta^{5}} \Psi=0
$$

where $\varepsilon$ is a smallness parameter. In the case of $\varepsilon=0$, (15) reduces identically to (13). Due to the secularities included in the last term of (15), it could not be solved exactly, e.g. only trivial solutions (travelling waves) were found by classical Lie group method; for a correct solution we rather have to rely on a perturbation method, in which the secularities embedded in the perturbed term are separated such that the equations in each order of $\varepsilon$ are secular. In the following we introduce the transformation $\eta=\zeta-\vartheta s$ to recast (15) into

$$
-\vartheta \frac{\mathrm{d} \Psi}{\mathrm{d} \eta}+A \Psi \frac{\mathrm{d} \Psi}{\mathrm{d} \eta}+\frac{B}{2} \frac{\mathrm{d}^{3} \Psi}{\mathrm{d} \eta^{3}}+\varepsilon \frac{\mathrm{d}^{5} \Psi}{\mathrm{d} \eta^{5}}=0 .
$$

In order to solve (16), we employ a simple method constructed by Watanabe and Jiang [23] for finding higher-order solitary wave solutions. Accordingly, we expand $\Psi$ and $\vartheta$ as well with respect to the smallness parameter $\varepsilon$ :

$$
\begin{aligned}
& \Psi=\psi_{0}+\varepsilon \psi_{1}+\varepsilon^{2} \psi_{2}+\varepsilon^{3} \psi_{3}+\ldots \\
& \vartheta=\vartheta_{0}+\varepsilon \vartheta_{1}+\varepsilon^{2} \vartheta_{2}+\varepsilon^{3} \vartheta_{3}+\ldots
\end{aligned}
$$

Substituting (17) into (16) and comparing the coefficients of like power in $\varepsilon$, one arrives at the following system of coupled ordinary differential equations:

$$
\begin{aligned}
& \varepsilon^{0}:-\vartheta_{0} \frac{\mathrm{d} \psi_{0}}{\mathrm{~d} \eta}+A \psi_{0} \frac{\mathrm{d} \psi_{0}}{\mathrm{~d} \eta}+\frac{B}{2} \frac{\mathrm{d}^{3} \psi_{0}}{\mathrm{~d} \eta^{3}}=0 \\
& \varepsilon^{1}: L \psi_{1}=\vartheta_{1} \frac{\mathrm{d} \psi_{0}}{\mathrm{~d} \eta}-\frac{\mathrm{d}^{5} \psi_{0}}{\mathrm{~d} \eta^{5}} \\
& \varepsilon^{2}: L \psi_{2}=\vartheta_{1} \frac{\mathrm{d} \psi_{1}}{\mathrm{~d} \eta}+\vartheta_{2} \frac{\mathrm{d} \psi_{0}}{\mathrm{~d} \eta}-A \psi_{1} \frac{\mathrm{d} \psi_{1}}{\mathrm{~d} \eta}-\frac{\mathrm{d}^{5} \psi_{1}}{\mathrm{~d} \eta^{5}} \\
& \varepsilon^{3}: L \psi_{3}=\vartheta_{3} \frac{\mathrm{d} \psi_{0}}{\mathrm{~d} \eta}+\vartheta_{2} \frac{\mathrm{d} \psi_{1}}{\mathrm{~d} \eta}+\vartheta_{1} \frac{\mathrm{d} \psi_{2}}{\mathrm{~d} \eta} \\
& -A \frac{\mathrm{d}\left(\psi_{1} \psi_{2}\right)}{\mathrm{d} \eta}-\frac{\mathrm{d}^{5} \psi_{2}}{\mathrm{~d} \eta^{5}}
\end{aligned}
$$

where the operator $L$ represents

$$
L=-\vartheta_{0} \frac{\mathrm{d}}{\mathrm{d} \eta}+A \psi_{0} \frac{\mathrm{d}}{\mathrm{d} \eta}+\frac{B}{2} \frac{\mathrm{d}^{3}}{\mathrm{~d} \eta^{3}}
$$

In fact, this operator is the single-variable version of the linearized $\mathrm{KdV}$ operator. To finally obtain the desired solutions, we solve (18) successively and subject them to the boundary conditions $\psi_{\mathrm{i}}=0 \quad(i=$ $0,1,2, \ldots), \frac{\mathrm{d} \psi_{i}}{\mathrm{~d} \zeta}=0$, and $\frac{\mathrm{d}^{2} \psi_{i}}{\mathrm{~d} \zeta^{2}}=0$ for $\eta \rightarrow \pm \infty$. To this aim, the first equation of (18) is fulfilled by a solitary wave solution of the form

$$
\psi_{0}=\psi_{m} \operatorname{sech}^{2}(D \eta)
$$

where the soliton amplitude $\psi_{m}$ and the soliton width $D^{-1}$ are

$$
\psi_{m}=\frac{3 \vartheta_{0}}{A} \text { and } D^{-1}=\sqrt{\frac{2 B}{\vartheta_{0}}} .
$$

Note that (20) is just a single-soliton solution of the $\mathrm{KdV}$ equation. In the next order of $\varepsilon$, i. e. substituting (20) into the second equation of (18), we obtain straightforwardly,

$$
\begin{aligned}
& L \psi_{1}= \\
& \operatorname{sech}(D \eta)^{4}\left(\frac{-1440 D^{5} \vartheta_{0}}{A}-\frac{144 B D^{3} \vartheta_{0}^{2}}{A^{2}}\right) \\
& \cdot \tanh (D \eta)+\operatorname{sech}(D \eta)^{6}\left(\frac{2160 D^{5} \vartheta_{0}}{A}\right. \\
& \left.+\frac{216 B D^{3} \vartheta_{0}^{2}}{A^{2}}+\frac{54 D \vartheta_{0}^{3}}{A^{2}}\right) \tanh (D \eta) \\
& +\operatorname{sech}(D \eta)^{2}\left(\frac{96 D^{5} \vartheta_{0}}{A}-\frac{6 D \vartheta_{0} \vartheta_{1}}{A}\right) \tanh (D \eta)
\end{aligned}
$$

(21) is a third-order linear differential equation associated with inhomogeneous terms on the right-hand side. The homogeneous equation, $L \psi_{1}=0$, satisfying the boundary conditions, has a solution that is proportional to $\operatorname{sech}^{2}(D \eta) \tanh (D \eta)$. Let us assume here a solution of the above equation of the form

$$
\psi_{1}=\mu_{1} \operatorname{sech}^{2}(D \eta)+\mu_{2} \operatorname{sech}^{4}(D \eta)
$$

with $\mu_{1}$ and $\mu_{2}$ representing constants, which can readily be proved by re-substitution. Then, it is easily to observe that the coefficient of $\operatorname{sech}^{2}(D \eta)$ on the lefthand side cancels out and $L \psi_{1}$ is expressed in terms of 
$\operatorname{sech}^{4}(D \eta)$ and $\operatorname{sech}^{6}(D \eta)$. In that case, it will be obviously that the coefficient of $\operatorname{sech}^{2}(D \eta)$ on the righthand side should vanish, which leads to the first order correction of the velocity:

$$
\vartheta_{1}=\frac{4 \vartheta_{0}^{2}}{B^{2}}
$$

Finally, the coefficients in the ansatz (22) can be found to constitute the solution

$$
\psi_{1}=\frac{-30 \vartheta_{0}^{2}}{A B^{2}} \operatorname{sech}^{2}(D \eta)+\frac{45 \vartheta_{0}^{2}}{A B^{2}} \operatorname{sech}^{4}(D \eta) .
$$

In order to evaluate next orders of $\varepsilon$, we introduce (20), (23), and (24) into the last equations of (18), and after some simple mathematical manipulations one can get

$$
\begin{aligned}
\vartheta_{2}= & 0, \\
\psi_{2}= & \frac{90 \vartheta_{0}^{3}}{A B^{4}} \operatorname{sech}^{2}(D \eta) \\
& -\frac{1395 \vartheta_{0}^{3}}{A B^{4}} \operatorname{sech}^{4}(D \eta) \\
& +\frac{1395 \vartheta_{0}^{3}}{A B^{4}} \operatorname{sech}^{6}(D \eta) . \\
\vartheta_{3}= & 0, \\
\psi_{3}= & \frac{-3708 \vartheta_{0}^{4}}{A B^{6}} \operatorname{sech}^{2}(D \eta) \\
& +\frac{31554 \vartheta_{0}^{4}}{A B^{6}} \operatorname{sech}^{4}(D \eta) \\
& -\frac{99324 \vartheta_{0}^{4}}{A B^{6}} \operatorname{sech}^{6}(D \eta) \\
& +\frac{74493 \vartheta_{0}^{4}}{A B^{6}} \operatorname{sech}^{8}(D \eta) .
\end{aligned}
$$

Combining now (20), (22), (23), (25), and (26), we obtain the solution of the perturbed KdV equation. This solution is expressed by power series of lowerorder solutions thus eliminating secularities, and the wave velocity depends on $\varepsilon$ only to the first-order, while the shape (profile of potential) of solitary wave depends on all orders of $\varepsilon$.

\section{Results and Discussion}

Nonlinear DIAWs in an unmagnetized, weakly inhomogeneous, weakly coupled, collisionless plasma have been investigated. To make our result physically

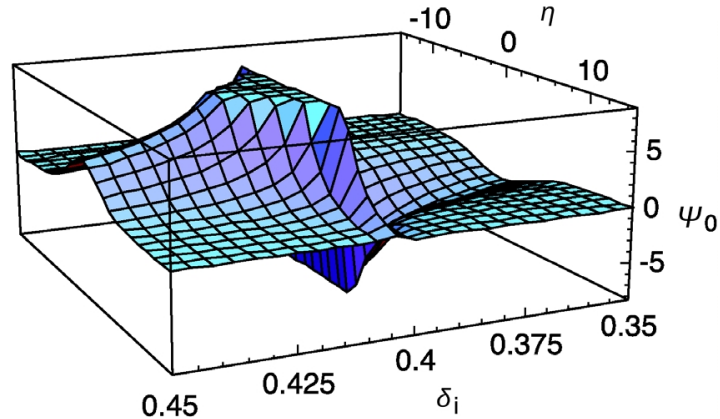

Fig. 1. Effect of variations of the ion density $\delta_{\mathrm{i}}$ on the amplitude and width of compressive and rarefactive solitons for $n_{\mathrm{e}}^{(0)}=1, \delta_{\mathrm{n}}=0.1, \delta_{\mathrm{p}}=0.5, Z_{\mathrm{n}}^{(0)}=2, Z_{\mathrm{p}}^{(0)}=1, \vartheta_{0}=0.2$, and $\lambda_{0}=1.2$.

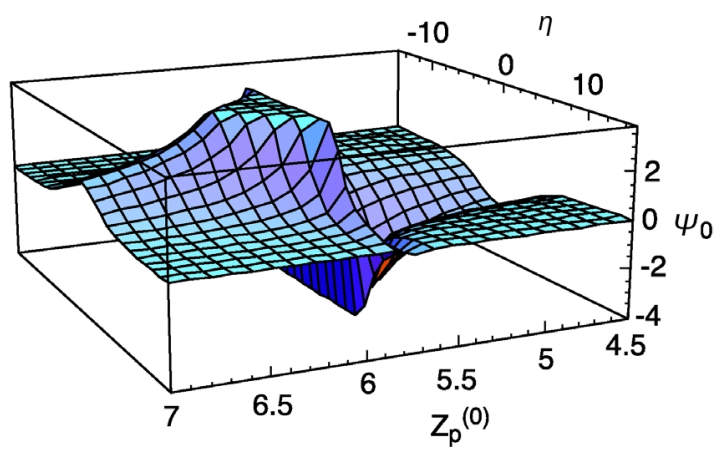

Fig. 2. Effect of variations of unperturbed positive dust grain charge $Z_{\mathrm{p}}^{(0)}$ on the amplitude and width of compressive and rarefactive solitons for $n_{\mathrm{e}}^{(0)}=1, \delta_{\mathrm{n}}=0.1, \delta_{\mathrm{p}}=0.5, Z_{\mathrm{n}}^{(0)}=2$, $\vartheta_{0}=0.4$, and $\lambda_{0}=1.2$.

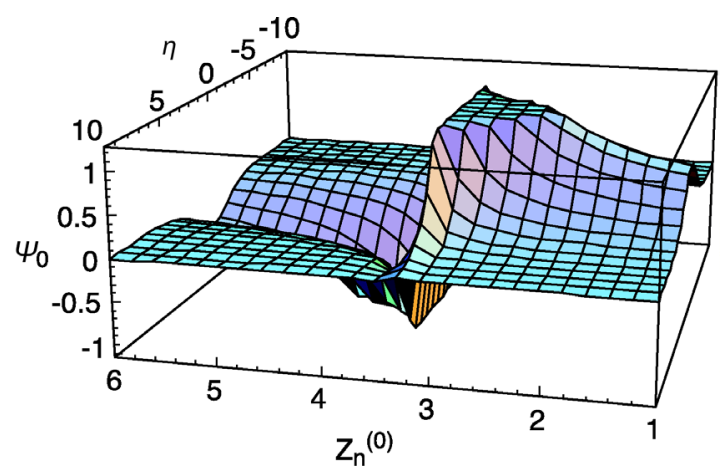

Fig. 3. Effect of variations of unperturbed negative dust grain charge number $Z_{\mathrm{n}}^{(0)}$ on the amplitude and width of compressive and rarefactive solitons for $n_{\mathrm{e}}^{(0)}=1, \delta_{\mathrm{n}}=0.1, \delta_{\mathrm{p}}=0.5$, $Z_{\mathrm{p}}^{(0)}=1, \vartheta_{0}=0.2$, and $\lambda_{0}=1.2$.

relevant, numerical calculations were performed referring to typical mesospheric dusty plasma parameters 


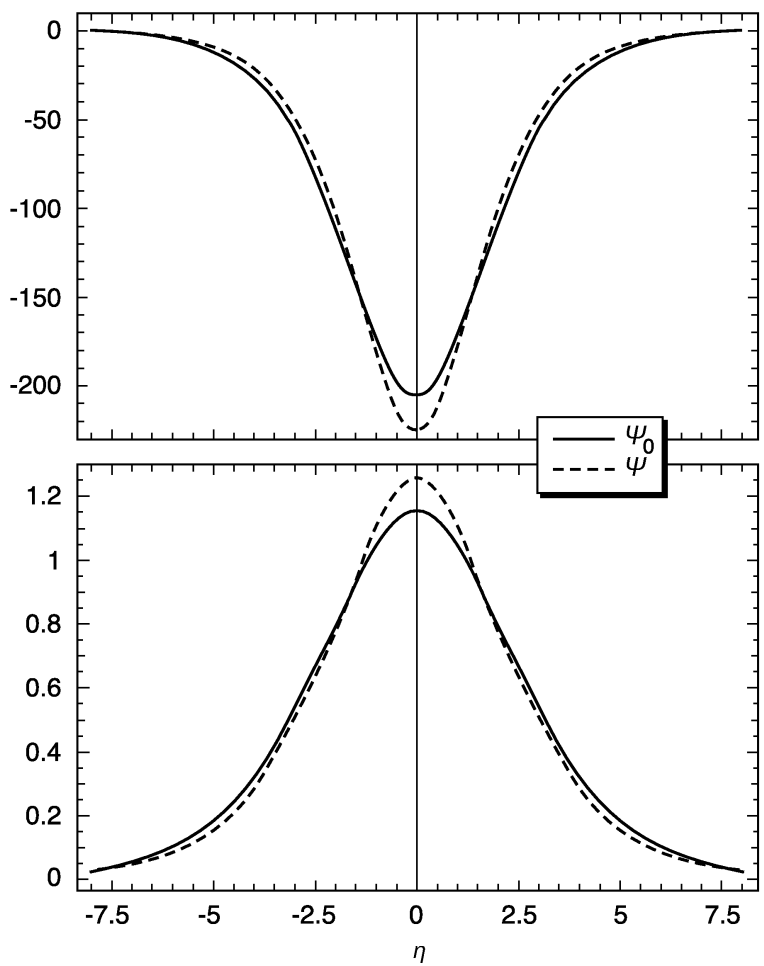

Fig. 4. Effect of higher-order dispersion on the soliton formation as apparent from comparing the lowest-order soliton potential $\psi_{0}$ with the higher-order soliton potential $\Psi$ for (a) $\delta_{\mathrm{i}}=0.4$ (rarefactive soliton) and (b) $\delta_{\mathrm{i}}=0.75$ (compressive soliton) depending on the travelling wave coordinate $\eta$. Here $n_{\mathrm{e}}^{(0)}=1, \delta_{\mathrm{n}}=0.1, \delta_{\mathrm{p}}=0.5, Z_{\mathrm{n}}^{(0)}=2, Z_{\mathrm{p}}^{(0)}=1, \varepsilon=0.09$, $\vartheta_{0}=0.7$, and $\lambda_{0}=1.2$ was assumed.

as given in [24]: $n_{\mathrm{i}}^{(0)}=10^{3} \mathrm{Cm}^{-3}, n_{\mathrm{e}}^{(0)}=10^{3} \mathrm{Cm}^{-3}$, $n_{\mathrm{p}}=500 \mathrm{Cm}^{-3}, n_{\mathrm{n}}=0.1 \mathrm{Cm}^{-3}, Z_{\mathrm{p}}^{(0)}=1, Z_{\mathrm{n}}^{(0)}=2$, $T_{\mathrm{i}}=0.01 \mathrm{eV}, T_{\mathrm{e}}=1 \mathrm{eV}, r_{\mathrm{d}}=20 \cdot 10^{-9} \mathrm{~m}, J=5 \cdot 10^{18}$ and $Y=0.1$.

We note that our dusty plasma model admits the coexistence of both rarefactive and compressive solitons, i. e. the amplitude of $\mathrm{KdV}$ solitons can be positive $(A>0)$ or negative $(A<0)$. For instance, we found from Figures 1 and 2 that the rarefactive soliton (width and amplitude) increases with enhancing the ion density $\delta_{\mathrm{i}}$ and unperturbed positive dust grain charge $Z_{\mathrm{p}}^{(0)}$ while the compressive soliton profile decreases, this means that the compressive soliton amplitude in inhomogeneous plasma will decreases as the wave propagates from low- to high-density region, while the rarefactive amplitude behaves the opposite way. In other words, the increase of the unperturbed negative dust grain charge number $Z_{\mathrm{n}}^{(0)}$ increases compressive soli-

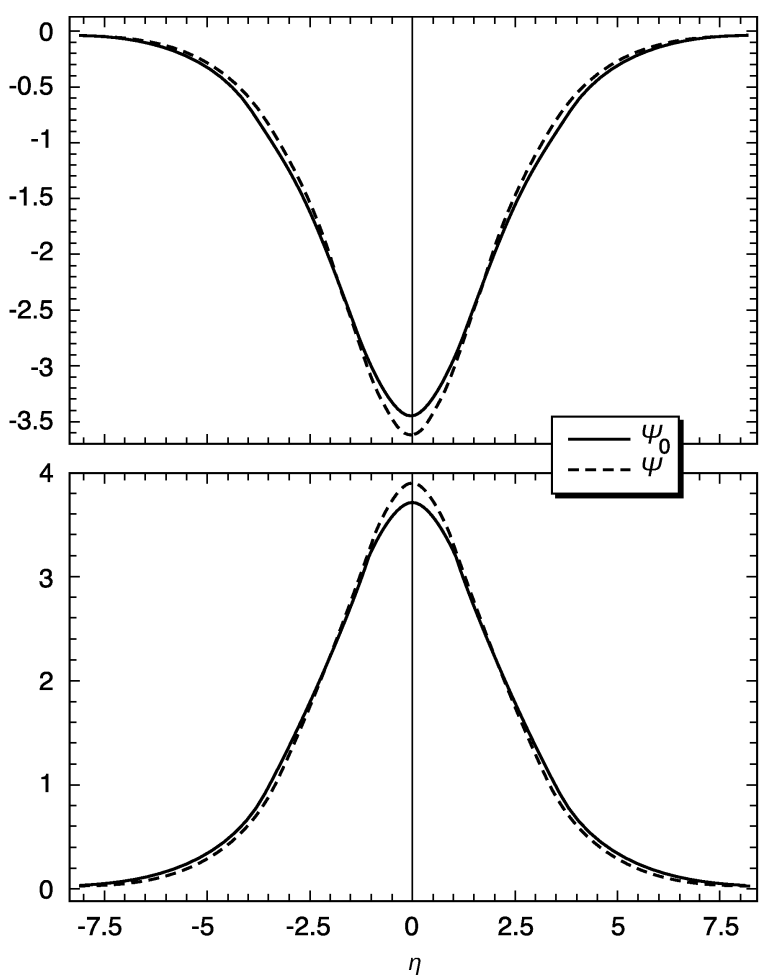

Fig. 5. Effect of higher-order dispersion on the soliton formation as apparent from comparing the lowest-order soliton potential $\psi_{0}$ with the higher-order soliton potential $\Psi$ for (a) $Z_{\mathrm{p}}^{(0)}=3$ (rarefactive soliton) and (b) $Z_{\mathrm{p}}^{(0)}=6$ (compressive soliton) depending on the travelling wave coordinate $\eta$. Here $n_{\mathrm{e}}^{(0)}=1, \delta_{\mathrm{i}}=0.3, \delta_{\mathrm{n}}=0.1, \delta_{\mathrm{p}}=0.5, \varepsilon=0.09, \vartheta_{0}=0.7$, and $\lambda_{0}=1.2$ was assumed.

ton (width and amplitude) and decreases the rarefactive soliton profile as displayed in Figure 3.

Since our objectives was to study the effect of higher-order dispersion on the formation of solitary waves, we specifically elucidated to what extent the higher-order solution modifies the soliton amplitude and width.

Moreover, the contribution of higher-order correction $\Psi$ for different values of ion density $\delta_{\mathrm{i}}$, unperturbed positive dust grain charge $Z_{\mathrm{p}}^{(0)}$, and unperturbed negative dust grain charge number $Z_{\mathrm{n}}^{(0)}$ are shown in Figures 4-6. Finally in Figure 7, the dependence of higher-order dispersion contribution on the parameter $\varepsilon$ is demonstrated for compressive and rarefactive solitons.

These results show that the ion density $\delta_{i}$, unperturbed negative dust grain charge number $Z_{\mathrm{n}}^{(0)}$, and unperturbed positive dust grain charge $Z_{\mathrm{p}}^{(0)}$ as well as the 


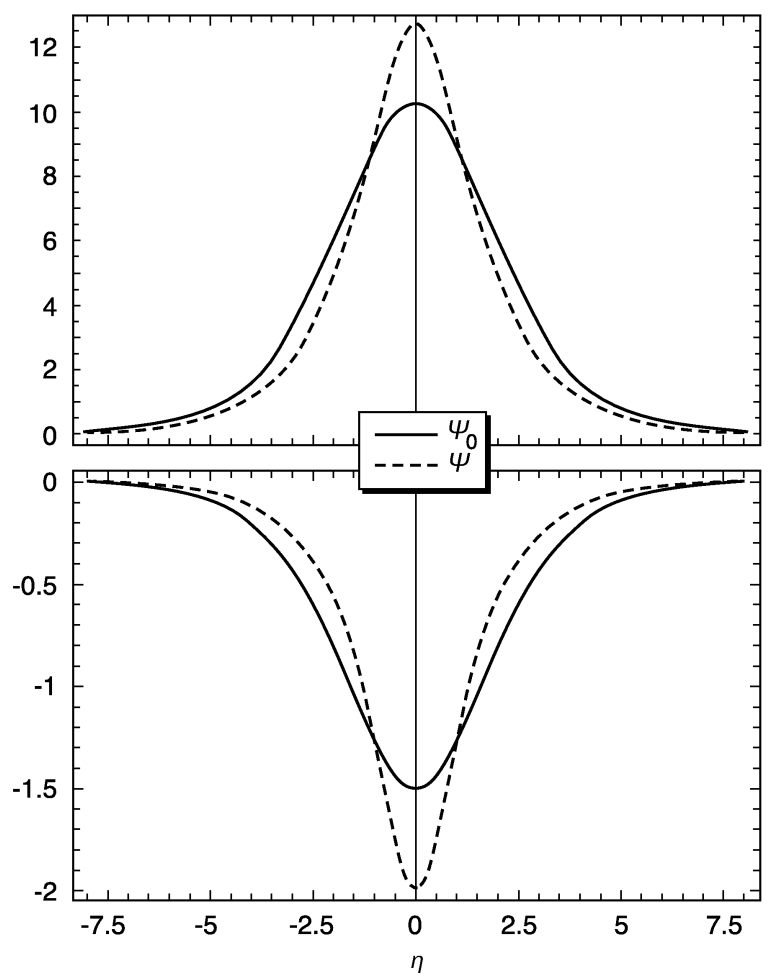

Fig. 6. Effect of higher-order dispersion on the soliton formation as apparent from comparing the lowest-order soliton potential $\psi_{0}$ with the higher-order soliton potential $\Psi$ for $a$ $Z_{\mathrm{n}}^{(0)}=1$ (compressive soliton) and $b-Z_{\mathrm{n}}^{(0)}=4$ (rarefactive soliton) depending on the travelling wave coordinate $\eta$. Here $n_{\mathrm{e}}^{(0)}=1, \delta_{\mathrm{i}}=0.88, \delta_{\mathrm{n}}=0.1, \delta_{\mathrm{p}}=0.5, Z_{\mathrm{p}}^{(0)}=1, \varepsilon=0.09$, $\vartheta_{0}=0.7$, and $\lambda_{0}=1.2$ was assumed.

higher-order dispersion modify significantly the properties of dust ion acoustic waves (DIAWs).

\section{Conclusion}

Though most of the pertinent literature on higherorder solutions in dusty plasma physics deals with homogeneous dusty plasma, in nature we will encounter dusty plasmas containing of a weakly inhomogeneous, weakly coupled, collisionless, and unmagnetized dusty plasma. In our theoretical treatment, we assumed that the phase velocity of DIAWs is much smaller (larger) than the electron thermal speed (ion and dust thermal speed). Hence, the electron number density perturbation associated with DIAWs is represented by Maxwellian distribution, while the ion number density perturbation can be measured from continuity equation. The reductive perturbation technique has been

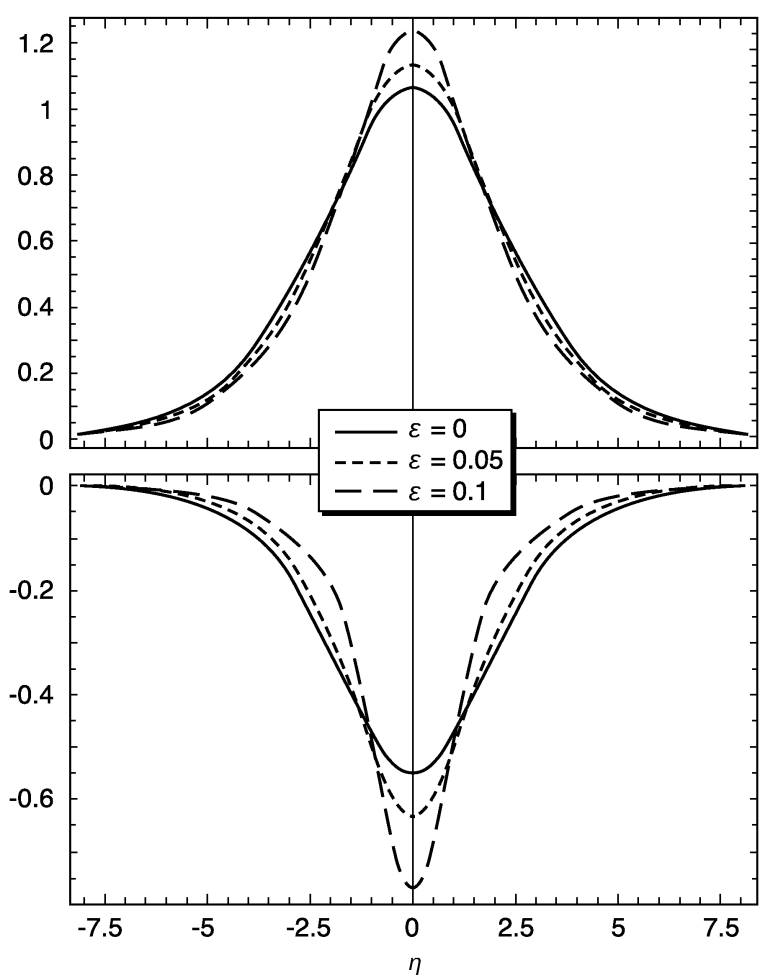

Fig. 7. The dependance of higher-order dispersion on the parameter $\varepsilon$ for (a) $Z_{\mathrm{n}}^{(0)}=2$ (compressive soliton) and (b) $Z_{\mathrm{n}}^{(0)}=5$ (rarefactive soliton) depending on the travelling wave coordinate $\eta$. Here $n_{\mathrm{e}}^{(0)}=1, \delta_{\mathrm{i}}=0.88, \delta_{\mathrm{n}}=0.1$, $\delta_{\mathrm{p}}=0.5, Z_{\mathrm{p}}^{(0)}=1, \vartheta_{0}=0.2$, and $\lambda_{0}=1.2$.

used to reduce the basic set of fluid equations to the well-known $\mathrm{KdV}$ equation. Interestingly, we observed that the presence of an additional dust component in our fluid model does not only modify the base properties of solitary potential structures, but also arises two different potential profiles, namely compressive and rarefactive, which is a completely new feature in dusty plasma [12,13]. It is emphasized that the amplitude and width of DIAWs solitons as well as the range of parameters, in which these solitons can exist, are significantly sensitive to the ion density $\delta_{\mathrm{i}}$, unperturbed negative dust grain charge number $Z_{\mathrm{n}}^{(0)}$, and unperturbed positive dust grain charge $Z_{\mathrm{p}}^{(0)}$. It was demonstrated that an enhancement of all these parameters can lead to a substantial modification of the DIAWs amplitude and width. Further, we have presented a simple method for obtaining higher-order solitary wave solutions by expanding a wave velocity as well as a wave amplitude. The velocity correction appears only in the first-order 
equation. This fact suggests that the velocity of a solitary wave is mainly determined by the nonlinear term, but depends weakly on the dispersive effect. So, we confirmed that the higher-order solution agrees with the numerical solution of the original equation [25]. In other words, the consideration of higher-order approximation was found to increase the amplitude and to decrease the width of DIAWs solitons. Finally, the contribution of higher-order dispersion depends mainly on the parameter $\varepsilon$ in additional to the ion density $\delta_{\mathrm{i}}$, unperturbed negative dust grain charge number $Z_{\mathrm{n}}^{(0)}$, and unperturbed positive dust grain charge $Z_{\mathrm{p}}^{(0)}$. We inject to that the analytical model demonstrated here can provide a useful basis for the interpretation of recent observations of solitary wave in dusty plasma environments. For example, the results presented may be applicable to dusty plasma existing in polar mesosphere region.

\section{Appendix}

$$
\begin{aligned}
& P_{1}=\frac{\pi r_{\mathrm{d}}^{2} J Y}{Z_{\mathrm{p}}^{(0)} \omega_{\mathrm{pi}}}, \quad P_{2}=\frac{Z_{\mathrm{p}} e^{2}}{r_{\mathrm{d}} K_{\mathrm{B}} T_{\mathrm{e}}}, \\
& P_{3}=\frac{\pi r_{\mathrm{d}}^{2} n_{\mathrm{e}}^{(0)}}{Z_{\mathrm{p}}^{(0)} \omega_{\mathrm{pi}}}\left(\frac{8 K_{\mathrm{B}} T_{\mathrm{e}}}{\pi m_{\mathrm{e}}}\right)^{\frac{1}{2}}, \\
& N_{1}=\frac{\pi r_{\mathrm{d}}^{2} n_{\mathrm{e}}^{(0)}}{Z_{\mathrm{n}}^{(0)} \omega_{\mathrm{pi}}}\left(\frac{8 K_{\mathrm{B}} T_{\mathrm{e}}}{\pi m_{\mathrm{e}}}\right)^{\frac{1}{2}}, \\
& N_{2}=\frac{\pi r_{\mathrm{d}}^{2} n_{\mathrm{e}}^{(0)}}{Z_{\mathrm{n}}^{(0)} \omega_{\mathrm{pi}}}\left(\frac{8 K_{\mathrm{B}} T_{\mathrm{i}}}{\pi m_{\mathrm{i}}}\right)^{\frac{1}{2}},
\end{aligned}
$$

[1] F. Verheest, Waves in Dusty Space Plasmas, Kluwer, Dordrecht 2000.

[2] P. K. Shukla and A. A. Mamun, Introduction to Dusty plasma Physics, Institute of Physics, Bristol 2002.

[3] R. N. Carlile, S. Geha, J. F. O'Hanlon, and J. C. Stewart, Phys. Lett. 59, 1167 (1991).

[4] P. K. Shukla and V.P. Siline, Physica Scr. 3, 508 (1992).

[5] A. Barkan, R. L. Merlino, and N. D’Angelo, Phys. Plasmas 2, 3563 (1995).

[6] R. Bharuthram and P. K. Shukla, Planet. Space Sci. 40, 973 (1992).

[7] N. D'Angelo, Planet. Space Sci. 42, 507 (1994).

[8] A. Mamun and P. K. Shukla, Phys. Plasmas 9, 1468 (2002).

$$
\begin{aligned}
& N_{3}=-\frac{e^{2} Z_{\mathrm{n}}^{(0)}}{C K_{\mathrm{B}} T_{\mathrm{e}}}, \quad N_{4}=\frac{e^{2} Z_{\mathrm{n}}^{(0)}}{C K_{\mathrm{B}} T_{\mathrm{i}}}, \\
& F_{1}\left(u_{0}\right)=\left(\frac{\sqrt{\pi}}{4 u_{0}}\right)\left(1+2 u_{0}^{2}\right) \operatorname{erf}\left(u_{0}\right)+\frac{1}{2} \exp \left(u_{0}^{2}\right) \text {, } \\
& F_{2}\left(u_{0}\right)=\left(\frac{\sqrt{\pi}}{2 u_{0}}\right) \operatorname{erf}\left(u_{0}\right), \quad \tilde{\lambda}_{0}=\lambda_{0}-u_{\mathrm{i}}^{(0)}, \\
& P_{4}=\frac{P_{3}\left(1+P_{2} Z_{\mathrm{p}}^{(0)}\right)}{P_{2}\left(P_{1}+P_{3} E_{1}\right)}, \\
& N_{5}=\left\{-N_{1}\left(1+N_{3} Z_{\mathrm{n}}^{(0)}\right) F_{1}\left(u_{0}\right)\right. \\
& \left.+N_{2} \delta_{\mathrm{i}}\left[+F_{2}\left(u_{0}\right) N_{4} Z_{\mathrm{n}}^{(0)}\right] \lambda_{0}^{-2}\right\} \\
& \text {. }\left\{N_{1} N_{3} E_{1}-N_{2} N_{4} \delta_{\mathrm{i}} F_{2}\left(u_{0}\right)\right\}^{-1}, \\
& P_{5}=\frac{P_{3} P_{4}}{\left(P_{1}+P_{3} E_{1}\right)}, \quad E_{1}=1+\phi^{(0)} \text {, } \\
& N_{6}=\frac{\left[F_{1}\left(u_{0}\right)+F_{2}\left(u_{0}\right) N_{4} Z_{\mathrm{n}}^{(0)}\right] N_{2}}{N_{1} N_{3} E_{1}-N_{2} N_{4} \delta_{\mathrm{i}} F_{2}\left(u_{0}\right)}, \\
& N_{7}=\frac{\left(-N_{1} N_{3}+N_{2} N_{4} \delta_{\mathrm{i}} F_{2}\left(u_{0}\right) \lambda_{0}^{-2}\right) N_{5}}{N_{1} N_{3} E_{1}-N_{2} N_{4} \delta_{\mathrm{i}} F_{2}\left(u_{0}\right)}, \\
& N_{8}=\frac{-N_{1}\left(1+N_{3} Z_{\mathrm{n}}^{(0)}\right)}{N_{1} N_{3} E_{1}-N_{2} N_{4} \delta_{\mathrm{i}} F_{2}\left(u_{0}\right)}, \\
& D_{1}=1-Z_{\mathrm{n}}^{(0)} \delta_{\mathrm{n}} N_{6} \text {, } \\
& D_{2}=\frac{3 \delta_{\mathrm{i}} D_{1}}{\lambda_{0}^{4}}=-2 Z_{\mathrm{n}}^{(0)} \delta_{\mathrm{n}} N_{7}+2 Z_{\mathrm{p}}^{(0)} \delta_{\mathrm{p}} P_{5} \text {. }
\end{aligned}
$$

[9] A. A. Mamun and P. K. Shukla, Geophys. Res. Lett. 29, 1870 (2002).

[10] Y. Nakamura, T. Odagiri, and I. Tsukabayashi, Plasma Phys. Control. Fusion 39, 105 (1997); Y. Nakamura and I. Tsukabayashi, Phys. Rev. Lett. 52, 2356 (1984).

[11] T. S. Gill, H. Kaur, and N. S. Saini, Pramana - J. Phys. 66, 1049 (2006).

[12] J.-J. Li and J. X. Ma, Physics Lett. A 318, 133 (2003).

[13] S. A. El-Wakil, M. A. Zahran, E. K. El-Shewy, and A. E. Mowafy, Phys. Scr. 74, 503 (2006).

[14] A.E. Mowafy, E. K. El-Shewy, W. M. Moslem, M. A. Zahran, Phys. Plasmas 15, 073708 (2008).

[15] S. K. El-Labany J. Plasma Phys. 54, 295 (1995).

[16] S. A. Elwakil, E. K. El-Shewy, and M. A. Zahran, Chaos, Solitons, and Fractals 22, 13 (2004). 
[17] W. F. El-Taibany and W. M. Moslem, J. Plasma Phys. 12, 032307 (2005).

[18] E. K. El-Shewy, Chaos, Solitons, and Fractals 26, 1073 (2005).

[19] S. A. Elwakil, M. T. Attia, M. A. Zahran, E. K. ElShewy, and H. G. Abdelwahed, Z. Naturforsch. 61a, 316 (2006).

[20] S. K. Elwakil, E. K. El-Shewy, and R. Sabry, Int. J. Nonlinear Sci. Numer. Simul. 5, 403 (2004).
[21] E. K. El-Shewy, Chaos, Solitons, and Fractals 34, 628 (2007).

[22] N. Asano, Prog. Theor. Phys. Suppl. 55, 52 (1974).

[23] S. Watanabe and B. Jiang, Phys Fluids B 5, 409 (1993).

[24] A. M. Zadorozhny, Adv. Space Res. 28, 1059 (2001).

[25] M. J. Ablowitz and H. Segur, Solitons and the inverse scattering transform. Society for Industrial and Applied, Philadelphia 1981. 